\title{
Enhanced photodegradation of di(2-ethyl)hexyl phthalate by Molecularly Imprinted titanium dioxide
}

\author{
Yu Shi $^{1}$, Fang-yan Chen*1, Li-na $\mathrm{He}^{1}$, Yu-bin Tang ${ }^{1,2}$ \\ ${ }^{1}$ School of Environmental and Chemical Engineering, Jiangsu University of Science and Technology, Zhenjiang, China \\ ${ }^{2}$ Zhangjiagang Industrial Technology Research Institute, Jiangsu University of Science and Technology, Zhangjiagang, China
}

\begin{abstract}
To enhance the photocatalytic degradation efficiency and selectivity of di(2-ethyl)hexyl phthalate (DEHP), molecularly imprinted $\mathrm{TiO}_{2}\left(\mathrm{MIP}-\mathrm{TiO}_{2}\right)$ was synthesized with di(2-ethylhexyl) phthalate as template molecule. The as-prepared $\mathrm{MIP}-\mathrm{TiO}_{2}$ was characterized by Field emission scanning electron microscopy (SEM), X-ray diffraction (XRD), Fourier infrared spectroscopy (FI-IR), UV-Visible diffuse reflection spectroscopy (UV-Vis) and photoluminescence spectroscopy (PL). The selective adsorption and photocatalytic activity of $\mathrm{MIP}_{-} \mathrm{TiO}_{2}$ for DEHP were studied. The results show that DEHP molecularly imprinted polymer was coated on the surface of $\mathrm{TiO}_{2}$. The light-response of $\mathrm{MIP}^{-\mathrm{TiO}_{2}}$ was broadened. The photo-generated electron/hole recombination on the surface of $\mathrm{TiO}_{2}$ decreased. The selective recognition to DEHP was improved. Compared with $\mathrm{TiO}_{2}, \mathrm{MIP}^{-\mathrm{TiO}_{2}}$ exhibited higher photocatalytic activity and selectivity to DEHP. The photocatalytic degradation efficiency of DEHP $(5 \mathrm{mg} / \mathrm{L})$ by $\mathrm{MIP}^{-\mathrm{TiO}_{2}}$ was $89 \%$. The photocatalytic degradation rate of DEHP by $\mathrm{MIP}^{-\mathrm{TiO}_{2}}$ is 1.5 times that by $\mathrm{TiO}_{2}$. $\mathrm{MIP}-\mathrm{TiO}_{2}$ could be reused.
\end{abstract}

\section{Introduction}

Phthalate (PAEs) is a kind of widely used plasticizer, which can increase the flexibility, plasticity and mechanical strength of plastics. PAEs combine with monomer molecule of plastic via hydrogen bonds or van der Waals forces, so they can easily migrate from plastic matrix into the environment [1-3]. As one of the most common PAEs, di(2-ethylhexyl) phthalate (DEHP) has been detected in soil, water and food around the world, and has been considered as an environmental priority pollutant in some countries [4,5]. DEHP has obvious male reproductive toxicity, which can cause testicular dysplasia, cryptorchidism, decreased sperm quality and potential threats to human liver metabolism [6-8]. Therefore, it is significant how to rapidly degrade DEHP in wastewater or environment.

The photocatalytic oxidation has attracted increasing attention due to their advantages of high efficiency and thorough degradation of pollutants [9]. $\mathrm{TiO}_{2}$ is a commonly used photocatalyst because of non-toxicity, high efficiency and optical stability $[10,11]$. However, $\mathrm{TiO}_{2}$ is lack of selectivity for photocatalytic degradation of organic pollutants, resulting in preferential degradation of other high-concentration organic substances coexisting in wastewater, while lowconcentration target pollutants that need to be removed are not effectively degraded $[12,13]$. Therefore, in order to improve the photocatalytic degradation efficiency of toxic organic pollutants with low concentrations in wastewater, it is of great significance to develop photocatalysts with high selectivity for the degradation of target pollutants. Molecularly imprinted polymers have well-matched pores and adsorption sites which can specifically recognize template molecules or molecules with similar structures, and have been widely used in chromatographic separation, solid phase extraction, sensors and other fields. In recent years, it has been found that the combination of photocatalytic technology and molecular imprinting can effectively improve the photocatalytic efficiency and selectivity of the catalyst for target pollutants [14-17]. A lot of molecularly imprinted $\mathrm{TiO}_{2}$ have been reported in degradation of some organic pollutants [14-18]. However, the molecularly imprinted $\mathrm{TiO}_{2}$ with DEHP as template molecule has not been reported. In addition, there are few reports on the effect mechanism of molecular imprinting modification on the photocatalytic performance of the catalyst.

In present work, a molecularly imprinted $\mathrm{TiO}_{2}$ was synthesized with DEHP as template molecule. The selectively photocatalytic degradation of DEHP by

*1Corresponding author: catchen1029@sohu.com 
molecularly imprinted $\mathrm{TiO}_{2}$ was investigated. The mechanism of molecular imprinting to improve photocatalytic performance was discussed, which provided basis for the development of photocatalysts with high selectivity.

\section{Experimental}

\subsection{Materials}

Titanium sulphate, ethylenediamine tetraacetic acid, urea, di(2-ethylhexyl) phthalate (DEHP), Dibutyl phthalate (DBP), dimethyl phthalate(DMP), methacrylic acid (MAA), ethylene glycol dimethacrylate (EGDMA), azobisisobutyronitrile (AIBN), acetic acid, absolute ethanol, and methanol were purchased from Sinopharm Chemical Reagent Co., Ltd. Titanium sulphate is of chemical grade. Methanol is chromatographically pure. The other reagents are of analytical grade.

\subsection{Preparation of $\mathrm{MIP}^{-\mathrm{TiO}_{2}}$}

MIP-TiO 2 was synthesized by microwave heating reaction method. Titanium sulfate (3.6 g) was dissolved in $250 \mathrm{~mL}$ of distilled water under vigrous agitation, and then ethylene diamine tetraacetic acid (5.6 g) and urea $(2.1 \mathrm{~g})$ were added into the solutions. After being fully stirred, the solution was transferred to the Teflon-lined reactor and heated by microwave for $8 \mathrm{~min}$. The resulting solid phase was centrifugally separated, and then washed with distilled water and anhydrous ethanol in turn. Finally, the solid was dried at $80^{\circ} \mathrm{C}$ in vacuum. White powder $\mathrm{TiO}_{2}$ was obtained.

DEHP ( $1 \mathrm{mmol})$ was dissolved in $80 \mathrm{~mL}$ of ethanol in a three-necked flask, MAA (4 mmol) was added into the flask, and then stirred at room temperature for $3 \mathrm{~h}$. $\mathrm{TiO}_{2}$ $(0.1 \mathrm{~g})$ was added into the flask and dispersed under ultrasound, afterwards, EGDMA (10 mmol) and AIBN (0.02 g) were successively added into the flask. The mixture in the flask reacted at $60{ }^{\circ} \mathrm{C}$ under the nitrogen protection for $18 \mathrm{~h}$. The product was washed with anhydrous ethanol, methanol/acetic acid and distilled water, respectively, dried in a vacuum at $60^{\circ} \mathrm{C}$, and the white powder $\mathrm{MIP}-\mathrm{TiO}_{2}$ was obtained.

\subsection{Photocatalytic experiments}

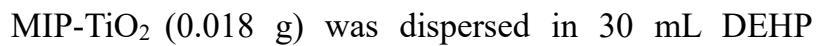
solution with the concentration of $5 \mathrm{mg} / \mathrm{L}$ in a glass reactor, and the suspension were magnetically stirred for $30 \mathrm{~min}$ in dark. Then, a $300 \mathrm{~W}$ ultraviolet lamp was turned on to illuminate the suspension. Samples were taken out for every $0.5 \mathrm{~h}$. The concentration of DEHP was determined by high performance liquid chromatography and the degradation efficiency was calculated as eq. (1).

$$
\eta=\frac{C_{0}-C_{t}}{C_{0}} \times 100 \%
$$

Where, $C_{0}$ and $C_{\mathrm{t}}$ are the initial concentration of DEHP and the residual concentration after being illuminated for $t \mathrm{~h}$, respectively.

\subsection{Determination of DEHP}

The concentration of DEHP was determined by high performance liquid chromatograph (LC-20A Ultrafast LC, Shimadzu Japan) with an Inertsil ODS-SP C18 column $(250 \mathrm{~mm} \times 4.6 \mathrm{~mm} \times 5 \mu \mathrm{m})$. The column temperature was $40^{\circ} \mathrm{C}$, and the mobile phase was methanol/water $(95: 5, \mathrm{~V} / \mathrm{V})$ with a flow rate of 0.5 $\mathrm{mL} / \mathrm{min}$. The wavelength of the UV detector was $228 \mathrm{~nm}$.

\section{Results and discussion}

\subsection{Characterization of $\mathrm{MIP}^{-\mathrm{TiO}_{2}}$}

\subsubsection{FT-IR spectra}

FT-IR spectra of MIP-TiO 2 and $\mathrm{TiO}_{2}$ are shown in Fig. 1. As given in spectrum of $\mathrm{TiO}_{2}$ (Fig.1a), the strong absorption peak that appears between 500 and $750 \mathrm{~cm}^{-1}$ corresponded to Ti-O bond. The absorption peaks at $3440 \mathrm{~cm}^{-1}$ and $1620 \mathrm{~cm}^{-1}$ was due to the stretching vibration and the bending vibration of the hydroxyl group from water molecular, respectively, indicating that bound moisture was adsorbed on the surface of $\mathrm{TiO}_{2}$ samples. In spectrum of $\mathrm{MIP}_{-} \mathrm{TiO}_{2}$ (Fig.1b), the adsorption band at $3443 \mathrm{~cm}^{-1}$ and $957 \mathrm{~cm}^{-1}$ are characteristic absorption peaks of $-\mathrm{OH}$. The peaks at $2972 \mathrm{~cm}^{-1}, 1461 \mathrm{~cm}^{-1}$ and $1382 \mathrm{~cm}^{-1}$ belonged to the stretching vibration of $-\mathrm{CH}_{2}$ in the polymer skeleton. The peak at $1259 \mathrm{~cm}^{-1}$ was attributed to the stretching vibration absorption of the ester bonds in the crosslinker EGDMA. The peaks at $1074 \mathrm{~cm}^{-1}$ and $1746 \mathrm{~cm}^{-1}$ was assigned to $\mathrm{C}=\mathrm{O}$ and $\mathrm{C}-\mathrm{O}$ bonds, respectively [20, 21]. It is concluded that crosslinking polymerization occurred on the surface of $\mathrm{TiO}_{2}$, and molecularly imprinted polymers were successfully synthesized.

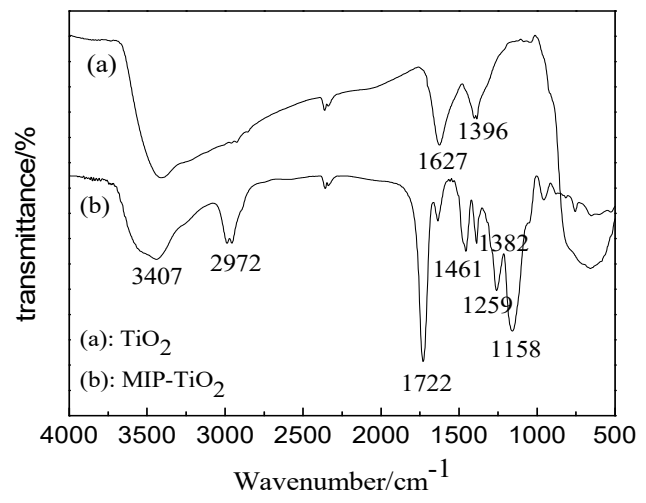

Fig. 1. FT-IR spectra of $\mathrm{TiO}_{2}$ (a) and $\mathrm{MIP}-\mathrm{TiO}_{2}$ (b)

\subsubsection{SEM}

The SEM images of MIP-TiO ${ }_{2}$ and $\mathrm{TiO}_{2}$ are shown in Fig. 2. As seen from Fig. 2(a), the $\mathrm{TiO}_{2}$ particles obtained are spherical, with particle sizes ranging from 200 to 300 $\mathrm{nm}$. The particle size distribution is relatively uniform, but the surface is relatively rough. In Fig. 2(b), the 
surface of the $\mathrm{MIP}-\mathrm{TiO}_{2}$ particles was smooth and flat, demonstrating that the molecularly imprinted polymer was successfully coated on the $\mathrm{TiO}_{2}$ surface.

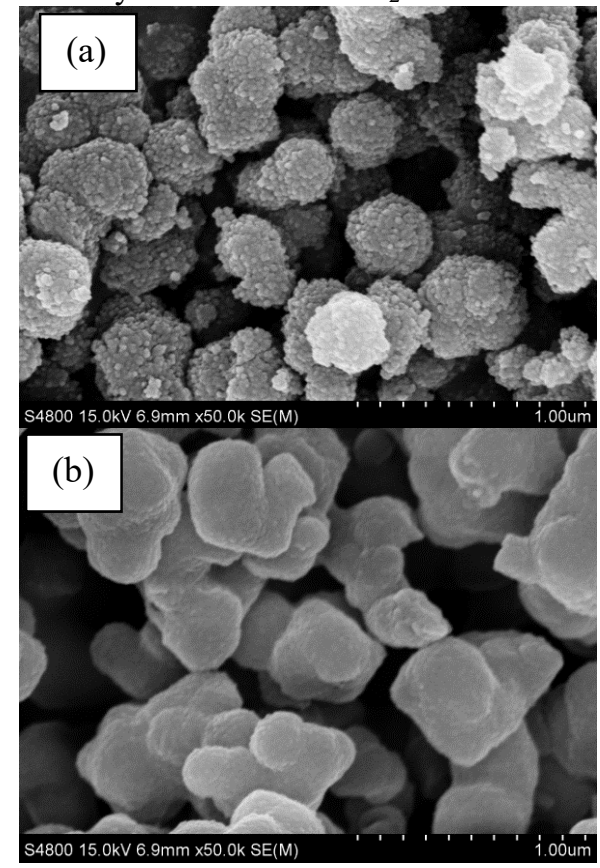

Fig. 2. SEM images of $\mathrm{TiO}_{2}$ (a) and $\mathrm{MIP}-\mathrm{TiO}_{2}$ (b).

\subsubsection{XRD analysis}

Fig. 3 shows the XRD patterns of $\mathrm{TiO}_{2}$ and $\mathrm{MIP}-\mathrm{TiO}_{2}$. It can be seen from Fig.3 (a) that the diffraction peaks at $2 \theta=25.3^{\circ}, 38.1^{\circ}, 48^{\circ}, 54.2^{\circ}, 55.4^{\circ}$ and $63^{\circ}$ can be assigned to (101), (004), (200), (105), (211) and (204) crystal planes of anatase $\mathrm{TiO}_{2}$, indicating that the sample prepared is anatase $\mathrm{TiO}_{2}$. The photocatalytic ability of anatase $\mathrm{TiO}_{2}$ is obviously stronger than that of rutile or amorphous $\mathrm{TiO}_{2}$, so, the $\mathrm{TiO}_{2}$ sample prepared by macrowave method has strong photocatalytic ability for degradation of target substances [19]. Fig. 3(b) shows that $\mathrm{MIP}-\mathrm{TiO}_{2}$ has the same diffraction peaks as $\mathrm{TiO}_{2}$. It is indicated that MIP-TiO 2 still maintains perfect anatase structure.

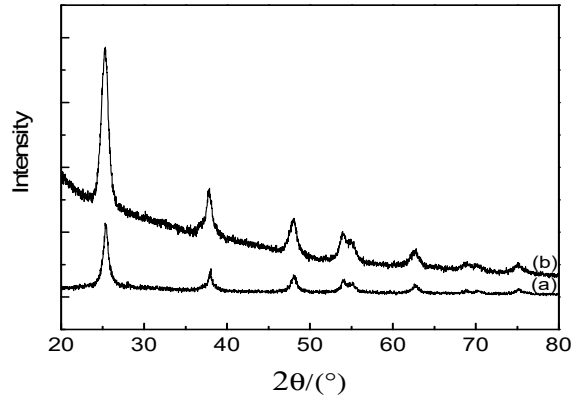

Fig. 3. XRD patterns of $\mathrm{TiO}_{2}$ (a) and $\mathrm{MIP}-\mathrm{TiO}_{2}$ (b)

\subsubsection{UV-vis diffuse reflection spectra}

UV-vis of $\mathrm{MIP}-\mathrm{TiO}_{2}$ and $\mathrm{TiO}_{2}$ are given in Fig.4. As shown in Fig. 4, compared with $\mathrm{TiO}_{2}, \mathrm{MIP}-\mathrm{TiO}_{2}$ has stronger light absorption in the entire visible region. This is because the introduction of reactive groups to the surface of $\mathrm{TiO}_{2}$ during molecularly imprinted polymerization improves the light absorption performance of the catalyst [16]. The band gaps energy of catalysts can be estimated by eq. (2) [22].

$$
E_{g}=\frac{h C}{\lambda_{g}}
$$

Where, $E_{\mathrm{g}}$ is the band gap energy, $h$ is the Planck's constant, $C(\mathrm{~m} / \mathrm{s})$ is the speed of light, and $\lambda_{\mathrm{g}}$ is the absorption wavelength (nm).

According to eq. (2), the band gaps energy of $\mathrm{TiO}_{2}$ and $\mathrm{MIP}-\mathrm{TiO}_{2}$ was calculated as $3.04 \mathrm{eV}$ and $2.91 \mathrm{eV}$, respectively. $\mathrm{MIP}-\mathrm{TiO}_{2}$ has a narrow band gap, which can generate more electron-hole under light irradiation. It is suggested that $\mathrm{MIP}-\mathrm{TiO}_{2}$ would exhibit higher photocatalytic activity than $\mathrm{TiO}_{2}$.

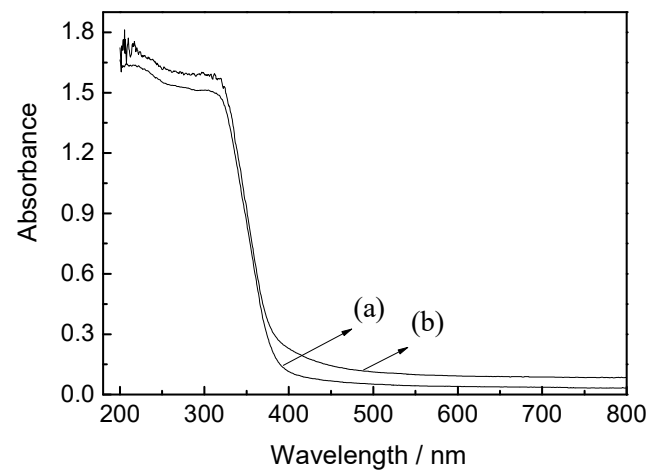

Fig. 4. UV-vis spectra of $\mathrm{TiO}_{2}$ (a) and $\mathrm{MIP}-\mathrm{TiO}_{2}$ (b)

\subsubsection{Photoluminescence (PL) spectra}

Photoluminescence is widely used to assess the recombination probability of electron-hole pairs. Fig.5 shows the PL spectra of $\mathrm{TiO}_{2}$ and $\mathrm{MIP}-\mathrm{TiO}_{2}$. It can be seen from Fig. 6 that the PL intensity of $\mathrm{MIP}_{-} \mathrm{TiO}_{2}$ decreased significantly, compared to $\mathrm{TiO}_{2}$. It is indicated that the molecularly imprinted polymer effectively inhibited the recombination of photo-generated electron/hole on the surface of $\mathrm{TiO}_{2}$, which would be beneficial to improve the photocatalytic activity of MIP$\mathrm{TiO}_{2}$.

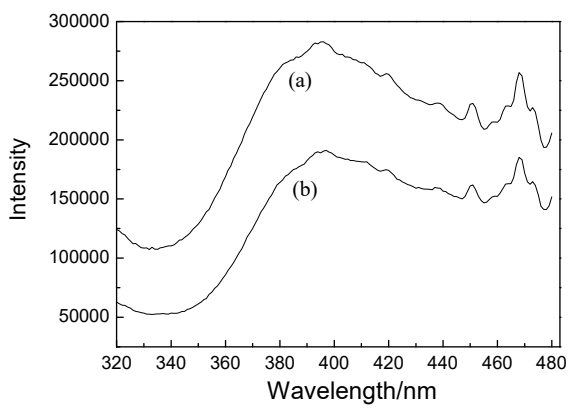

Fig. 5. $\mathrm{PL}$ spectra of $\mathrm{TiO}_{2}$ (a) and $\mathrm{MIP}-\mathrm{TiO}_{2}$ (b)

\subsection{Adsorption properties of $\mathrm{MIP}^{-\mathrm{TiO}_{2}}$ for DEHP}

The adsorption performance of the target pollutant on the catalyst directly affects the photocatalytic activity. In order to investigate the adsorption performance of MIP- 
$\mathrm{TiO}_{2}$ for $\mathrm{DEHP}$, the adsorption rate and adsorption selectivity of $\mathrm{MIP}^{-\mathrm{TiO}_{2}}$ to DEHP were determined.

\subsubsection{Adsorption rate curve of DEHP onto MIP-TiO}

The adsorption rate curve of DEHP onto MIP-TiO ${ }_{2}$ is depicted in Fig. 6. As shown in Fig.6, the adsorption of DEHP onto $\mathrm{TiO}_{2}$ and $\mathrm{MIP}-\mathrm{TiO}_{2}$ reaches adsorption equilibrium after $30 \mathrm{~min}$, but the equilibrium adsorption capacity of DEHP onto MIP- $\mathrm{TiO}_{2}$ is significantly higher than that onto $\mathrm{TiO}_{2}$. This is because there is a binding site on the molecularly imprinted layer on the surface of MIP-TiO ${ }_{2}$ that matches the target molecule DEHP, which greatly increases the adsorption capacity of DEHP. It is implied that $\mathrm{MIP}-\mathrm{TiO}_{2}$ have stronger photocatalytic degradation ability.

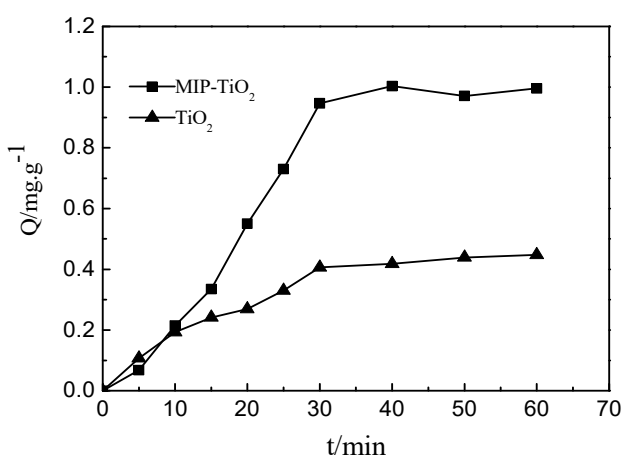

Fig. 6. curves of Adsorption rate $\mathrm{MIP}-\mathrm{TiO}_{2}$ and $\mathrm{TiO}_{2}$ for $\mathrm{DEHP}$

\subsubsection{Adsorption selectivity of MIP-TiO 2 for DEHP}

In order to investigate the selective recognition ability of MIP-TiO ${ }_{2}$ to DEHP, MIP-TiO ${ }_{2}$ and $\mathrm{TiO}_{2}$ were used to adsorb DEHP and its analogs DBP and DMP with initial concentration of $5 \mathrm{mg} / \mathrm{L}$, and the equilibrium adsorption amount is given in Fig. 7. As shown in Fig. 7, the adsorption capacities of DEHP and its analogs DBP and DMP on $\mathrm{TiO}_{2}$ is lower than that on MIP-TiO difference in adsorption capacity of the three pollutants is very small, indicating that $\mathrm{TiO}_{2}$ has no selectivity to DEHP. While, the adsorption capacity of DEHP onto MIP-TiO ${ }_{2}$ is significantly larger than that of DBP and DMP, and is obviously higher than that onto $\mathrm{TiO}_{2}$, indicating that $\mathrm{MIP}^{-\mathrm{TiO}_{2}}$ can specifically recognize DEHP molecules. This specific recognition not only can enhance the adsorption capacity of DEHP on the catalyst surface and thus enhance the photocatalytic effect; but also can greatly enhance photocatalytic selectivity of MIP-TiO 2 to the target molecule DEHP.

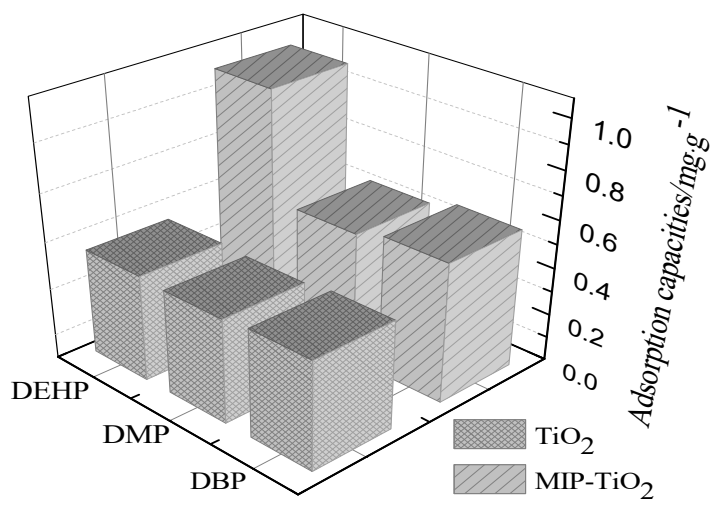

Fig. 7. Adsorption of DEHP and its analogues onto ${\mathrm{MIP}-\mathrm{TiO}_{2}}_{2}$ The imprinting factor $(\alpha)$ and the adsorption selectivity coefficient $(\beta)$, which can be calculated by formulas (4) and (5), respectively [23], are usually used to evaluate adsorption selectivity of molecularly imprinted polymer to template molecules.

$$
\begin{gathered}
k=\frac{Q_{e}}{C_{e}} \\
\alpha=\frac{K_{M I P-T i O 2}}{K_{T i O 2}} \\
\beta=\frac{\alpha_{\mathrm{DEHP}}}{\alpha_{i}}
\end{gathered}
$$

Where, $Q_{\mathrm{e}}$ is equilibrium adsorption capacity $(\mathrm{mg} / \mathrm{g})$ of the target molecules onto the two catalysts, $C_{\mathrm{e}}$ is the concentration of DEHP $(\mathrm{mg} / \mathrm{L})$ at the adsorption equilibrium, $\alpha_{\mathrm{DEHP}}$ is the imprinting factor of DEHP, and $\alpha_{\mathrm{i}}$ is the imprinting factor of DBP or DMP.

According to formula (4) and (5), the imprinted factor of $\mathrm{MIP}^{-\mathrm{TiO}_{2}}$ to DEHP, DBP and DMP and selectivity coefficients of MIP-TiO ${ }_{2}$ to DEHP were calculated and listed in table 1 . It can be seen from table 1 that the imprinting factor of $\mathrm{MIP}^{-\mathrm{TiO}_{2}}$ to DEHP is significantly larger than that to DBP and DMP, and the selectivity coefficients of MIP-TiO ${ }_{2}$ to DBP and DMP is 2.92 and 3.01 , respectively.

Table 1. Adsorption selectivity of the $\mathrm{MIP}-\mathrm{TiO}_{2}$

\begin{tabular}{ccccccc}
\hline \multirow{2}{*}{ Analytes } & $\begin{array}{c}Q_{\mathrm{MIP}-} \\
\text { TiO2 }(\mathrm{mg} / \mathrm{g})\end{array}$ & $Q_{\mathrm{TiO} 2}(\mathrm{mg} / \mathrm{g})$ & $K_{\mathrm{MIP}-\mathrm{TiO} 2}(\mathrm{~L} / \mathrm{g})$ & $K_{\mathrm{TiO} 2}(\mathrm{~L} / \mathrm{g})$ & $\alpha$ & $\beta$ \\
\hline DBP & 0.585 & 0.457 & 0.119 & 0.092 & 1.30 & 2.92 \\
DMP & 0.548 & 0.439 & 0.111 & 0.088 & 1.26 & 3.01 \\
DEHP & 0.995 & 0.448 & 0.449 & 0.118 & 3.79 & - \\
\hline
\end{tabular}

\subsection{Photocatalytic degradation of DEHP}

The photocatalytic degradation efficiencies of DEHP are given in Fig.8. As seen in Fig. 8, the photodegradation efficiency of DEHP by $\mathrm{MIP}^{-\mathrm{TiO}_{2}}$ is significantly higher than that $\mathrm{TiO}_{2}$. Within 180min, DEHP can be degraded $89 \%$ by $\mathrm{MIP}-\mathrm{TiO}_{2}$.

The photodegradation process of pullutants is generally simulated by the first-order kinetics as eq.(6) [24]. 


$$
\ln \left(\frac{c_{0}}{c_{t}}\right)=k t
$$

Where, $C_{0}$ is initial concentration of pollutant in solution. $C_{\mathrm{t}}$ is the concentration of pollutant solution at time t. $k$ can be regarded as an apparent rate constant, the photocatalytic degradation ability of the catalyst can be known by $k$ value.

The experimental data shown in Fig. 8 was fitted by the first-order kinetics model and the results are shown in table 2 .

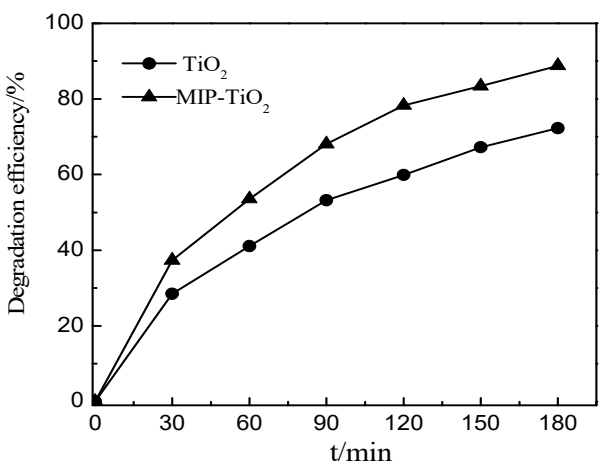

Fig. 8. Photocatalytic degradation rate of DEHP by $\mathrm{MiP}_{-} \mathrm{TiO}_{2}$ and $\mathrm{TiO}_{2}$

Table 2. Kinetics parameters for photocatalytic degradation of DEHP

\begin{tabular}{ccc}
\hline Catalyst & $k\left(\mathrm{~min}^{-1}\right)$ & Correlation coefficient $R^{2}$ \\
\hline $\mathrm{MIP}_{-\mathrm{TiO}}$ & 0.0116 & 0.9917 \\
$\mathrm{TiO}_{2}$ & 0.0079 & 0.979 \\
\hline
\end{tabular}

As given in table 2 , the correlation coefficients more than 0.97 suggested that photocatalytic degradation of DEHP by MIP-TiO 2 and $\mathrm{TiO}_{2}$ can be simulated by the first-order kinetics. Moreover, the value of $k$ indicated the degradation rate of DEHP by MIP-TiO 2 is 1.5 times that by $\mathrm{TiO}_{2}$.

\subsection{Photocatalytic selectivity of MIP-TiO2 for DEHP}

In order to investigate the selectivity of photocatalytic degradation of $\mathrm{MIP}-\mathrm{TiO}_{2}$ for DEHP, the photocatalytic degradation reactions of DEHP, DBP and DMP by MIP$\mathrm{TiO}_{2}$ and $\mathrm{TiO}_{2}$ were carried out under the same conditions. The results were shown in Fig. 9. As given in Fig. 9 that the degradation efficiency of DBP by MIP$\mathrm{TiO}_{2}$ and $\mathrm{TiO}_{2}$ is basically similar to each other, and only $67 \%$. At the preliminary stage, the degradation efficiency of DMP by MIP-TiO 2 was slightly higher than that by $\mathrm{TiO}_{2}$, but after $3 \mathrm{~h}$, the degradation efficiency of DMP by MIP-TiO ${ }_{2}$ was identical to the degradation efficiency of DMP by $\mathrm{TiO}_{2}$, which was about $60 \%$. These results indicate that $\mathrm{MIP}^{-\mathrm{TiO}_{2}}$ has no selectivity for photocatalysis of DBP and DMP. The degradation efficiency of DEHP by MIP-TiO 2 is $89 \%$, far higher than that of DBP (67\%) and DMP (61\%).

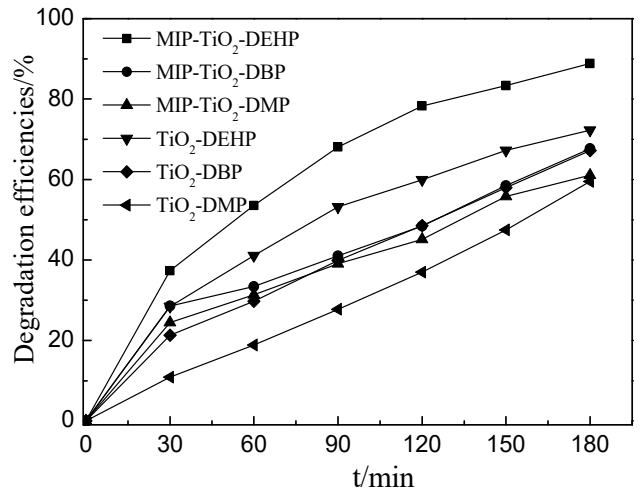

Fig. 9. The degradation rate of $\mathrm{MIP}-\mathrm{TiO}_{2}$ over different target

The data shown in Fig. 9 was fitted by equation (6) to obtain the photocatalytic degradation rate constants of DEHP, DBP and DMP by MIP-TiO 2 and $\mathrm{TiO}_{2}$ and the results are listed in table 3 .

The photocatalytic selectivity of $\mathrm{MIP}-\mathrm{TiO}_{2}$ to the template molecule can be expressed by a selectivity coefficient. The selectivity coefficient can be calculated according to eq. (7) and the results are also listed in table 3.

$$
\begin{aligned}
& \alpha=\frac{R_{\text {template }}}{R_{\text {ana } \log u \text { e }}} \\
& R=\frac{k_{M}}{k_{T}}
\end{aligned}
$$

Where, $R_{\text {template }}$ is ratio of photocatalytic degradation rate constant of template molecule by $\mathrm{MIP}^{-\mathrm{TiO}_{2}}$ and $\mathrm{TiO}_{2}$, calculated by formula (8). $R_{\text {analogue }}$ is ratio of photocatalytic degradation rate constant of analogs DBP and DMP by MIP-TiO 2 and $\mathrm{TiO}_{2}$.

As listed in table 3 , the selectivity coefficient of DEHP relative to DBP and DMP is 1.52 and 1.44 , respectively. It is indicated that $\mathrm{MIP}-\mathrm{TiO}_{2}$ exhibits excellently photocatalytic selectivity to DEHP, and can degrade DEHP preferentially.

Table 3. Kinetics parameters and Selectivity coefficient of $\mathrm{MIP}_{-} \mathrm{TiO}_{2}$

\begin{tabular}{ccccccccc}
\hline \multirow{2}{*}{ Parameters } & \multicolumn{3}{c}{${\mathrm{MIP}-\mathrm{TiO}_{2}}$} & & \multicolumn{3}{c}{$\mathrm{TiO}_{2}$} \\
\cline { 2 - 4 } \cline { 6 - 8 } & DEHP & DBP & DMP & & DEHP & DBP & DMP \\
\hline$k\left(\mathrm{~min}^{-1}\right)$ & 0.0116 & 0.0056 & 0.0049 & & 0.0079 & 0.0058 & 0.0048 \\
$R\left(k_{\mathrm{M}} / k_{\mathrm{T}}\right)$ & 1.47 & 0.97 & 1.02 & & - & - & - \\
$\alpha\left(R_{\text {target }} / R_{\text {non-target }}\right)$ & - & 1.52 & 1.44 & & - & - & - \\
\hline
\end{tabular}

To investigate the reutilization of photocatalyst MIP- 
separated, cleaned and dried, and then used to degrade $\operatorname{DEHP}(5 \mathrm{mg} / \mathrm{L})$ again. The procedure mentioned above was repeated 5 times, and the photocatalytic efficiency for every time is shown in Fig. 10.

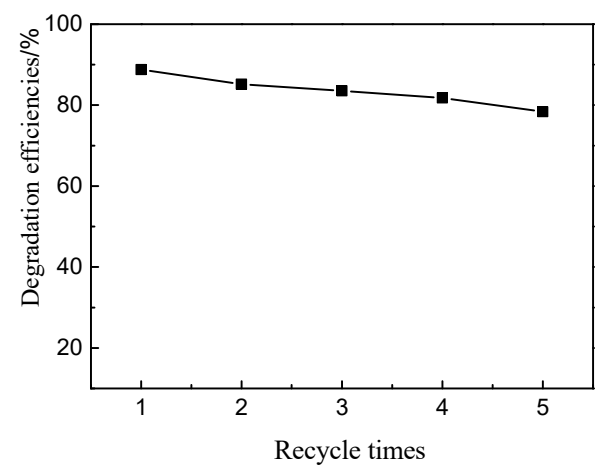

Fig. 10. Recycle of $\mathrm{MIP}-\mathrm{TiO}_{2}$

As seen in Fig. 10, the photocatalytic efficiency of DEHP is maintained between $88.8 \%$ and $78.4 \%$ during the reuse of $\mathrm{MIP}-\mathrm{TiO}_{2}$. Although the photocatalytic degradation efficiency of DEHP decreases slightly with the increasing in reuse of $\mathrm{MIP}-\mathrm{TiO}_{2}$, considering the photocatalytic efficiency decrease resulted from a certain loss of $\mathrm{MIP}-\mathrm{TiO}_{2}$ during solid-liquid separation, washing and drying, it is, therefore, concluded that $\mathrm{MIP}-\mathrm{TiO}_{2}$ is reusable.

\section{Conclusions}

MIP-TiO 2 was successfully synthesized with DEHP as template molecule, MAA as monomer and $\mathrm{TiO} 2$ as support. $\mathrm{MIP}-\mathrm{TiO}_{2}$ retained the same anatase structure as $\mathrm{TiO}_{2}$. Compared with $\mathrm{TiO}_{2}, \quad \mathrm{MIP}-\mathrm{TiO}_{2}$ exhibited enhanced light absorption, red-shifted absorption sideband and obviously specific recognition ability for DEHP. The imprinting factor of $\mathrm{MIP}^{-\mathrm{TiO}_{2}}$ is 3.29, and the selectivity coefficient of $\mathrm{MIP}-\mathrm{TiO}_{2}$ toward DMP and DBP is 3.01 and 2.92, respectively. The DEHP molecularly imprinted polymer on the surface of $\mathrm{TiO}_{2}$ effectively inhibited the photo-generated electron/hole recombination.

The photocatalytic degradation rate of DEP by MIP$\mathrm{TiO}_{2}$ is 1.5 times that of $\mathrm{TiO}_{2}$. The photocatalytic degradation efficiency of DEHP with concentration of 5 $\mathrm{mg} / \mathrm{L}$ was $89 \%$. MIP-TiO 2 showed significantly photocatalytic selectivity for DEHP with selectivity coefficient of 1.52 and 1.44 for DEHP to DBP and DMP, respectively. The photocatalytic efficiency of DEHP was maintained in the range of $88.8 \%$ to $78.4 \%$ during being reused for 5 times.

\section{Acknowledgements}

This work was financially supported by the science and technology support projects of Zhangjiagnag City, China (Grant No. ZKS1510)

\section{References}

1. B. Bagó, Y. Martín, G. Mejía, F. Broto-Puig, et al. Chemosphere, 59, 1191 (2005)

2. S. Huma, M. Najma, K. Hamayun, et al. J. Chromatogr. A., 1247, 125(2012)

3. T. J. Gray, S. D. Gangolli, Environ. Health Perspect., 65, 229(1986)

4. H. Fromme, T. Kuchler, T. Otto, et al. 36, 1429 (2002)

5. J. Wang, Y.M. Luo, Y. Teng, et al. Environ Pollut., 180, 265 (2013)

6. M.M Abdel, U.J Rivera, R. Ocampo-PÉREZ, et al. J. Environ. Manage., 109, 164 (2012)

7. M. Julinová, R. Slavik. J. Environ. Manage., 94, 13 (2012)

8. H. Wei, Q. Ning, X.Z. Kong, et al. Sci. Total Environ., 461, 672 (2013)

9. X.L. Liu, P. Lv, G.X. Yao, et al. Colloids Surf. A. 441, 420 (2014)

10. F. Deng, Y.X. Li, X.B. Luo, et al. Colloids Surf. A., 395, 183 (2012)

11. S. Anandan, N. Pugazhenthiran, T. Lana-Villarreal et al. Chem. Eng. J., 231, 182(2013)

12. K. Kabra, Chaudhary, R.L. Sawhney, et al. Ind. Eng. Chem. Res., 43, 7683 (2004)

13. X.Y. Tang, S.Y. Wang, Y. Yang, et al. Chem. Eng. J. 275, 198 (2015)

14. W.Q. Sun, R. Tian, W.G. Zheng, et al. Chinese J. Catal., 34, 1589 (2013)

15. H. Li, G. Li, Z.P. Li, et al. Appl. Surf. Sci., 264, 644(2013)

16. X.L. Liu, P. Lv, G.X. Yao, et al. Chem. Eng. J. 217, 398 (2013)

17. X. T. Shen, L. H. Zhu, N. Wang, et al. Catal. Today., 225, 164(2014)

18. Z. Q. Wang, X. Liu, W.Q. Li, et al. Ceram. Int., 40, 8863 (2014)

19. H. M. Zhao, Y. Chen, X. Quan, et al. Chin. Sci. Bull., 52, 1456 (2007)

20. B. Singh, N. Chauhan, V. Sharma. Int. Eng. Chem. Res., 50, 13742 (2011)

21. Y. Z. Yang, X. G. Liu, M. C. Guo, et al. Colloids Surf. A. 377, 379 (2011)

22. F. J. Benitez, F. J. Real, J. L. Acero, et al. J. Chem. Technol. Biotechnol., 84, 1186(2009)

23. Z. Yang, F. Y. Chen*, Y. B. Tang, S. L. Li, J. Chem. Soc. Pak., 37, 939 (2015)

24. L. Yang, L. E. Yu, M. B. Ray. Water Res., 42, 3480(2008) 